\title{
基于循环训练法的变压器漏油检测
}

\author{
铇伟超 ${ }^{1)}$, 顾理 ${ }^{1)}$, 何劲松 ${ }^{1}{ }^{*}$, 萠勇 ${ }^{2)}$, 刘玮 ${ }^{2)}$, 黄文礼 ${ }^{2)}$ \\ ${ }^{1)}$ (中国科学技术大学信息科学技术学院 合肥 230026) \\ 2) (安徽继远电网技术有限责任公司 合肥 230088) \\ (hjss@ustc.edu.cn)
}

\begin{abstract}
摘 要: 检查变压器是否存在漏油问题在维护电网安全与稳定方面具有重要价值. 地面是否存在因漏油而产生的油 污区域是判断变压器是否存在漏油问题的重要的依据. 油污区域的形状各异、所处的环境复杂以及光照阴影的影响 给漏油检测问题带来了挑战. 阴影是自然界中的一种普遍存在的物理现象, 对漏油检测的影响是不可避免的. 为了 消除阴影对漏油检测的影响, 提出一种循环训练方法. 通过直方图均衡化以增强困难样本油污和阴影之间的对比度, 循环地训练增强后的图像来减弱阴影的干扰，以提高查全率；同时通过引人负样本图像缓解误检问题，以提高查准 率. 文中使用变电站真实环境下采集的数据, 并以此构建了一个油污图像的数据集. 基于此数据集设计 8 种方案进行 对比实验. 实验结果表明, 与未使用所提方法的模型相比, 使用该方法的模型能够有效地消除光照阴影对漏油检测 的影响, 显著提高漏油检测精确度.
\end{abstract}

关键词: 变压器漏油检测; 循环训练; 困难样本; 负样本

中图法分类号: TP391.41 DOI: 10.3724/SP.J.1089.2021.18361

\section{Transformer Oil Leakage Detection Based on Loop Training Method}

\author{
Bao Weichao ${ }^{1)}, \mathrm{Gu} \mathrm{Li}^{1)}$, He Jinsong ${ }^{1 *}$, Kuai Yong ${ }^{2)}$, Liu Wei ${ }^{2)}$, and Huang Wenli ${ }^{2)}$ \\ 1) (School of Information Science and Technology, University of Science and Technology of China, Hefei 230026) \\ 2) (Anhui Jiyuan Electricity Grid Technical Co., Ltd., Hefei 230088)
}

\begin{abstract}
Inspecting whether the transformer has oil leakage problem is of great value in maintaining the safety and stability of the power grid. The oil stain on the ground is an important basis for judging whether the transformer leaks oil. The different shapes of the oil stain areas, complex background and the influence of shadow have brought challenges to the oil stain detection. Shadow is a ubiquitous physical phenomenon in nature, and the impact on oil stain detection is inevitable. In order to eliminate the influence of shadow, we propose a loop training method. The histogram equalization is adopted to enhance the contrast of the hard example between the oil stain area and the shadow, and the enhanced images are iteratively trained to reduce the interference of the shadow to improve the recall. At the same time, we introduce negative examples to alleviate the false detection of oil stain to improve the precision. The data are collected in the real environment of the substation. To validate the effectiveness of the proposed method, 8 schemes are designed for comparison experiments. Experimental results show that the models using the proposed method can effectively eliminate the influence of shadow on oil stain detection, and significantly improve the accuracy of oil stain detection.
\end{abstract}

收稿日期: 2020-05-06; 修回日期: 2020-12-19. 鲍伟超(1995-), 男, 硕士研究生, 主要研究方向为机器学习、目标检测; 顾理 (1975-), 男 硕士, 讲师, 主要研究方向为智能信息处理、模式识别; 何劲松(1967一), 男, 博士, 副教授, 硕士生导师, 论文通讯作 者, 主要研究方向为演化计算、模式识别; 萠勇(1988-), 男, 硕士, 主要研究方向为智能信息处理; 刘玮(1983一), 男, 学士, 主要研 究方向为智能信息处理; 黄文礼(1987一), 男, 硕士, 主要研究方向为智能信息处理. 
Key words: oil stain detection; loop training; hard example; negative example

电能是现代社会运作的核心能源. 电能的供 给如果发生故障, 将会导致巨大的经济损失. 变电 站是电力系统的核心, 在电力输配中起着关键性 的作用. 在变电站内各类设备中, 变压器的使用最 为广泛. 为了保证变压器长时间的正常运行, 需避 免变压器发生漏油现象 ${ }^{[1]}$. 一旦变压器发生漏油且 未被及时发现，导致持续漏油将会对电网的稳定 运行带来隐患 ${ }^{[2]}$. 因此, 巡检人员需要预防变压器 发生漏油情况. 如果发生渗漏, 相关的维护管理人 员必须尽快发现漏油点并进行维修, 将因漏油产 生的危害降到最低.

近年来, 视频监控技术 ${ }^{[3]}$ 在变电站安全巡检中 得到了广泛的关注. 由于视频监控图像具有直观、 方便的特点, 将计算机视觉技术应用于电力设备 的在线监控, 发现设备运行过程中的故障, 可以减 少人力资源的消耗，保障巡检的质量.

目前, 深度学习算法在计算机视觉领域 ${ }^{[4-6]}$ 取 得了快速发展, 它可以从数据中自动学习, 提取感 兴趣的特征. 在深度学习兴起之后, 目标检测从手 工提取特征方法变成了基于卷积神经网络的特征 提取, 在行人检测 ${ }^{[7]}$ 、车辆检测 ${ }^{[8]}$ 、医学图像肿瘤 检测 ${ }^{[9]}$ 等方面得到了广泛的应用. 目标检测框架主 要有 2 类: 一类是以 $\mathrm{R}-\mathrm{CNN}^{[10]}$ 和 Faster $\mathrm{R}-\mathrm{CNN}^{[11]}$ 为代表的两段式目标检测器; 另一类是以 $\mathrm{YOLO}^{[12]}$ 和 $\mathrm{SSD}^{[13]}$ 为代表的一段式目标检测器. 两段式检测器具有较高的检测准确率，但其检测 速度不如一段式检测器. 一段式检测器采用的密 集采样候选区域的机制通常会存在正负样本不平 衡问题, 导致训练过程受到影响并降低最终的检 测准确率. 2017 年提出的焦点损失函数(focal loss, $\mathrm{FL})^{[14]}$ 为一段式目标检测器的正负样本不平衡问 题提供了新的解决方案.

检测变压器是否存在漏油问题, 直接的方法 是检测在变压器周围的地面上有无因漏油而形成 的油污区域. 然而, 区别于 $\mathrm{MSCOCO}^{[15]}$ 和 PASCAL VOC ${ }^{[16]}$ 等公共数据集中的目标, 油污目 标具有以下 2 个特点. 由于变压器不同的安装环境 以及绝缘油的流动性，使得图像中的油污区域呈 现出多种多样的形状. 此外, 油污一般呈现为黑色 区域，与光照产生的阴影极为相似. 阴影是自然界 中一种普遍存在的物理现象, 对油污检测的影响 是难以避免的. 阴影对油污检测的影响有 2 个方 面: 一方面, 阴影覆盖在油污上, 减小了油污与地
面背景之间的差异，对油污目标的信息造成一定 的损失与干扰，在识别过程中易漏掉一些油污区 域, 增加油污检测的难度; 另一方面, 背景上的阴 影和油污区域具有相似的颜色特征，阴影易被误 检为油污.

考虑检测准确率和检测速度，本文选用 RetinaNet ${ }^{[14]}$ 作为变压器漏油油污的基本检测模型. 针对上述漏油检测所面临的实际应用问题, 仅仅 通过调参训练模型的方法难以胜任油污目标的检 测与识别任务. 为消除光照阴影对油污检测的影 响, 提出一种循环训练方法, 它使模型能够针对现 有的难训练样本 (困难样本), 有针对性地学习其特 征, 提升模型的检测能力. 同时, 通过引入负样本 图像, 以缓解因阴影所造成误检的问题.

\section{RetinaNet 检测模型}

RetinaNet 的整体结构如图 1 所示, 它由 1 个 主干网络和 2 个特定功能的子网络组成. 其中, 主 干网络采用建立在 ResNet ${ }^{[17]}$ 之上的 $\mathrm{FPN}^{[18]}$. 综合 考虑细节信息低但语义信息丰富的高层特征, 以 及语义信息不足但细节信息高的低层特征, FPN 通 过自顶向下和横向连接的方式构建丰富的多尺度 特征金字塔，使得各尺度下的特征都有丰富的语 义信息. 金字塔共 5 层 $\left(P_{3} \sim P_{7}\right)$, 均有 256 个通道. 其中, $P_{3} \sim P_{5}$ 是经由中间层 $M_{3} \sim M_{5}$ 卷积得到, 中间 层 $M_{3} \sim M_{5}$ 是使用 ResNet 的残差层 $C_{3} \sim C_{5}$ 经过自顶 向下和横向连接的方式生成, $P_{6}$ 和 $P_{7}$ 分别在 $C_{5}$ 和 $P_{6}$ 的基础上进行核大小为 $3 \times 3$, 步长为 2 的卷积 得到.

2 个子网络分别为分类子网络和回归子网络, 子网络之间不共享参数, 其对主干网络的输出层

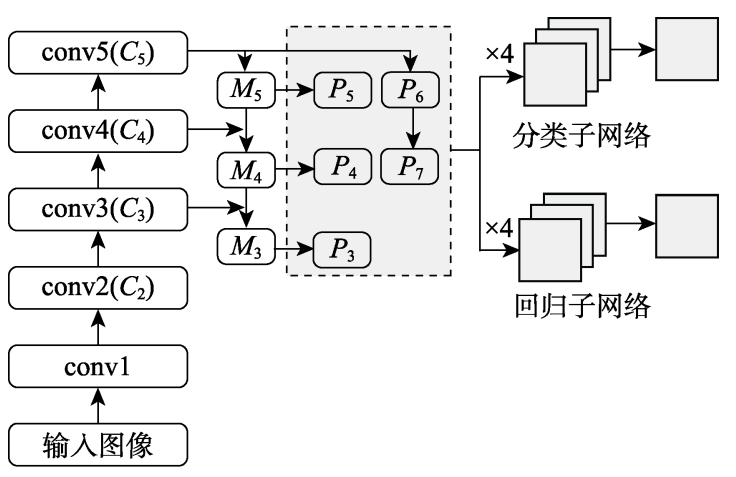

图 1 RetinaNet 整体结构 
$P_{3} \sim P_{7}$ 完成分类与回归任务, 并生成一系列的候选 框. 随后对这些候选框进行非极大值抑制(non maximum suppression, NMS), 去除同一目标重复 的候选框, 得到最终的预测框. 可以看出, 图 2a中 存在多个候选框.

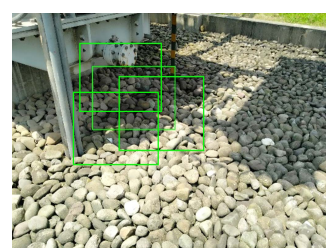

a. 未进行 NMS

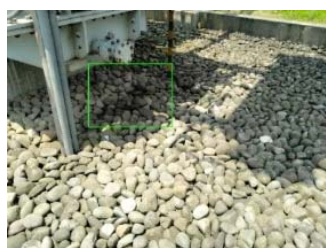

b. 进行 NMS 后
图 2 NMS 效果对比

$\mathrm{FL}$ 是在标准交叉熵损失函数 (cross entropy, $\mathrm{CE}$ )基础上修改得到的一种新型的损失函数，为一 段式目标检测器中样本类别不平衡问题提供了一 种新的解决思路. 二分类估计概率表示为

$$
p_{t}=\left\{\begin{array}{l}
p, \quad \text { if } y=1 \\
1-p, \text { otherwise }
\end{array} .\right.
$$

其中, $y$ 是真实标签, 表示标准 $\mathrm{CE}$ 损失函数, 即 $\mathrm{CE}\left(p_{t}\right)=-\log \left(p_{t}\right) . \mathrm{FL}$ 表示为

$$
\operatorname{FL}\left(p_{t}\right)=-\left(1-p_{t}\right)^{\gamma} \log \left(p_{t}\right) .
$$

通过引人调制因子 $\left(1-p_{t}\right)^{\gamma}$ ，每个样本可以获得不 同的损失，模型可以更多地集中在不易分类的样 本上.

\section{2 循环训练法}

采用图 3a 所示的常规训练法难以消除阴影对 油污检测的影响, 本文提出一种循环训练方法, 循 环地训练直方图均衡化后的困难样本, 循环训练 法流程如图 $3 b$ 所示.

困难样本的学习问题存在于目标检测 ${ }^{[19]}$ 和语 义分割 ${ }^{[20]}$ 中. 对困难样本进行挖掘学习可以加快 算法的收玫速度，提高模型学习的效果. 但是，对 于困难样本还没有明确的定义。在目标检测中，文 献[13]生成一系列候选区域，根据其损失值判定是 否为困难样本. 在本文的漏油检测问题中, 将困难 样本视为因光照产生的阴影对模型性能存在影响 的样本, 如该样本漏检或误检了油污区域. 在模型 的训练阶段, 通过对比网络的预测输出与真实标 签判断该样本是否存在误检或漏检的现象，从而 挖掘出困难样本.

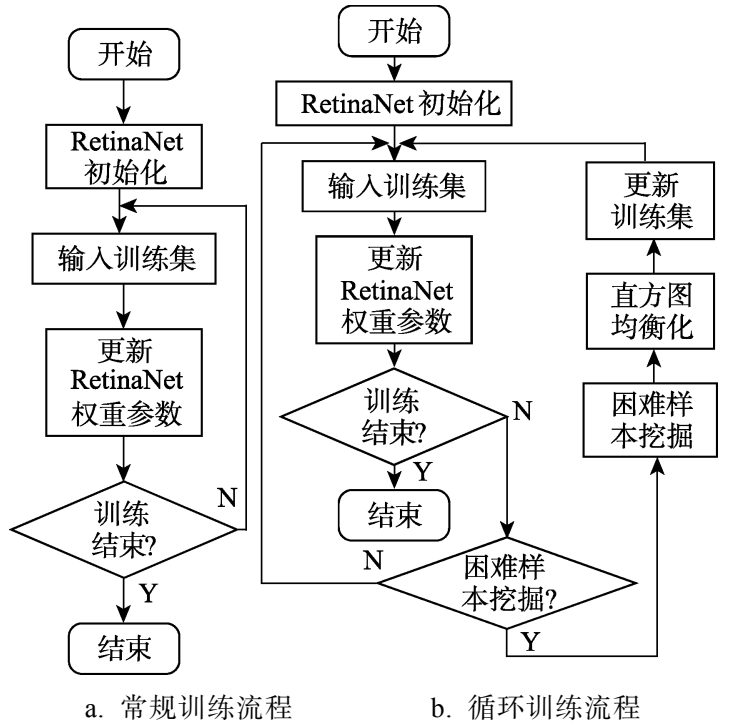

图 3 训练流程

直方图均衡化 ${ }^{[21]}$ 是一种增强图像对比度的方 法, 其主要思想是将一幅灰度图像的直方图分布 转化为近似均匀分布. 而对于三通道的 RGB 图像, 将其转换到 $\mathrm{YCrCb}$ 空间, 对 $Y$ 通道进行直方图均 衡化. 图 4 和图 5 所示为进行直方图均衡化后的图 像与原始图像的效果对比. 其中, 图 4 中直方图均 衡化操作增强了油污与阴影之间的对比度, 未引 人其他干扰因素. 而在图 5 的第 1 3 行中, 经过直 方图均衡化操作后反而削弱了较多的油污信息,

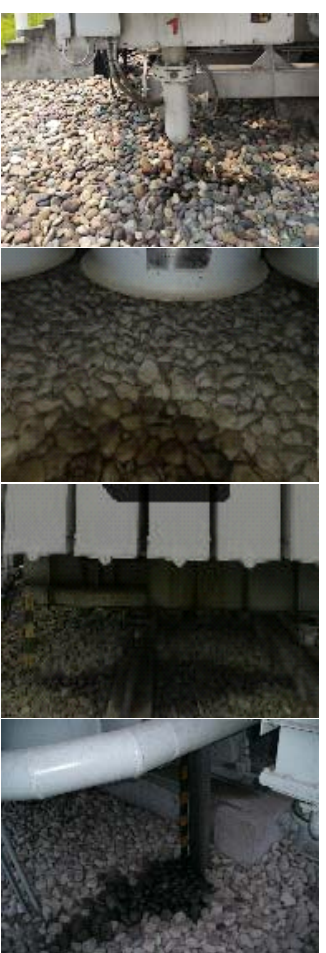

a. 原始图像

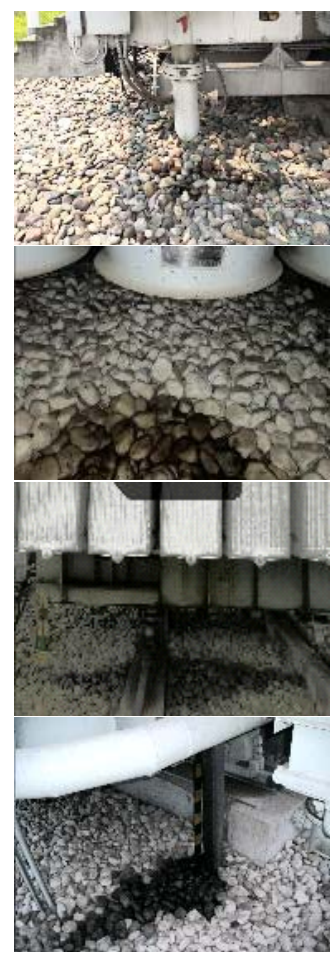

b. 直方图均衡化
图 4 直方图均衡化后效果对比(增强) 


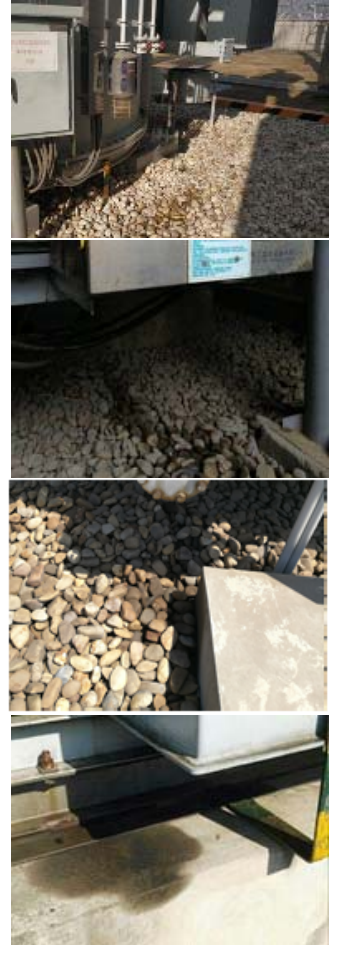

a. 原始图像

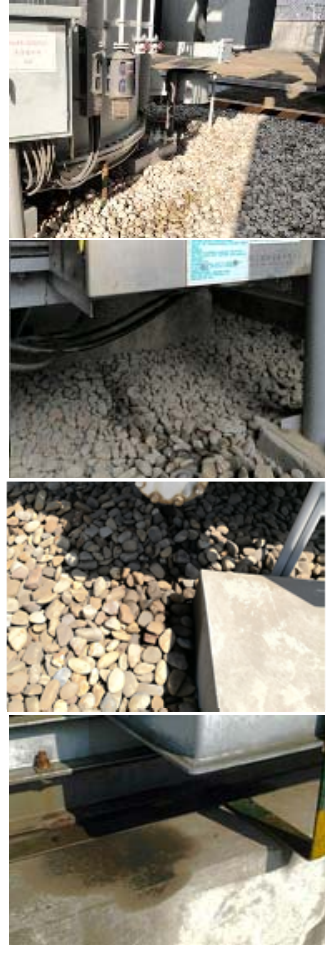

b. 直方图均衡化
图 5 直方图均衡化后效果对比(削弱)

并且引人了一些额外干扰, 如在图 5 第 4 行中的油 污区域与水泥地面几乎融为一体. 这些因素都对 油污检测模型的优化造成干扰. 而困难样本通常 代表难以检测的样本, 非困难样本通常容易检测. 对于非困难样本, 再对其进行直方图均衡化可能 会引人一些上文描述的干扰因素.

因此, 本文中通过困难样本挖掘方法挖掘出 困难样本，以避免引人一些干扰因素. 使用直方图 均衡化方法来增大油污和阴影之间的对比度, 以 突出图像中的油污区域; 循环地训练增强后的困 难样本, 以提高模型的泛化能力.

循环训练法的步骤如下:

Step1. RetinaNet 初始化.

Step2. 输人训练集, 迭代更新 RetinaNet 权重参数.

Step3. 若训练结束, 则算法结束并退出; 否则, 执行 下一步

Step4. 若网络训练次数大于或等于 $S$, 或者距上 一次困难样本挖掘后网络训练次数大于 $L$, 执行下一 步; 否则, 转 Step2.

Step5. 进行困难样本挖掘, 对困难样本直方图均 衡化后将其添加至训练集, 并更新训练集, 转 Step2.

在图像分类任务中，文献[22]利用数据增强技 术将原始图像进行裁剪, 并将裁剪后的图像与原 始图像一起输人模型中, 然后将它们的输出平均 作为模型的最终输出, 以提升模型的准确率. 因
此，本文给出一种应用于油污检测的综合测试法， 其测试流程如图 6a 所示.

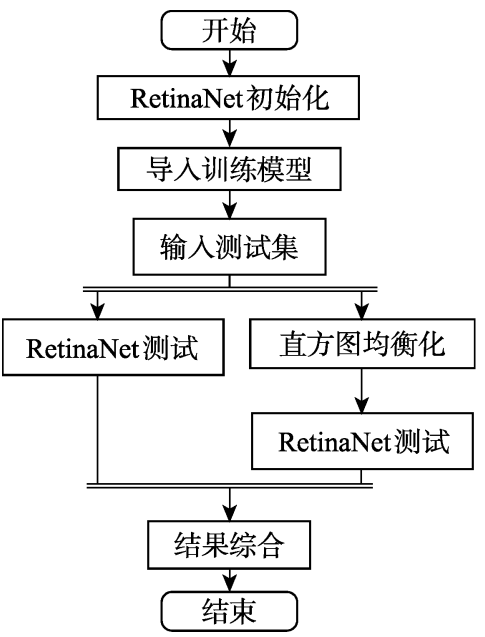

a. 常规测试流程

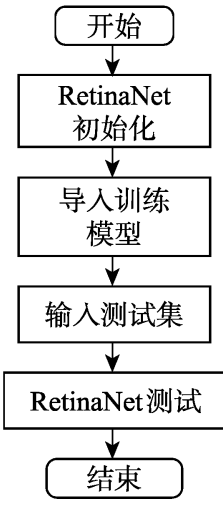

b. 综合测试流程
图 6 测试流程

与如图 $6 \mathrm{~b}$ 所示的常规测试流程相比，在测试 模型时, 综合测试法分别测试直方图均衡化增强 后的图像和原始图像，综合 2 次测试的结果并进行 NMS 后作为最终的测试结果. 其关键在于综合 2 次的检测结果, 即结合一系列的预测框, 并根据预 测框的分类置信度的大小以及 NMS 选择最优的预 测框. 图 7 所示为测试结果的综合过程. 图 7a 和图 7c 所示分别为原始图像和增强图像在 RetinaNet 上 的测试结果, 输出为油污目标候选框; 随后综合 2 次测试结果再用 NMS 去除多余的预测框, 从而获 得如图 $7 \mathrm{~d}$ 所示最终的测试结果.

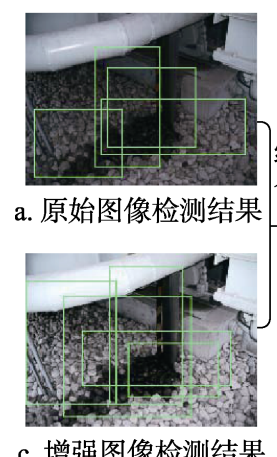

图 7 测试结果综合过程

\section{3 引入负样本图像}

在油污的实际检测中, 由于光照产生的阴影 与油污具有较高的相似性, 模型的分类子网络不 能正确区分阴影和油污，因此阴影区域容易被误 
检为油污. 因此, 提出通过训练负样本图像来解决 这个问题; 负样本图像是指不包含任务所检测目 标的图像. 在模型的训练过程中, 将原始训练集与 负样本图像共同训练模型. 原始训练集产生的候 选框根据其与真实标签的交并比值进行正负样本 的分配，而负样本图像产生的候选框都分配为负 样本. 本文根据误检图像的特点, 利用变电站视频 监控图像制作 50 幅不包含油污区域但包含光照阴 影的图像作为负样本来训练模型, 以增强模型对 阴影的敏感度，提高模型对阴影和油污的分类能 力. 图 8 所示为部分负样本图像.

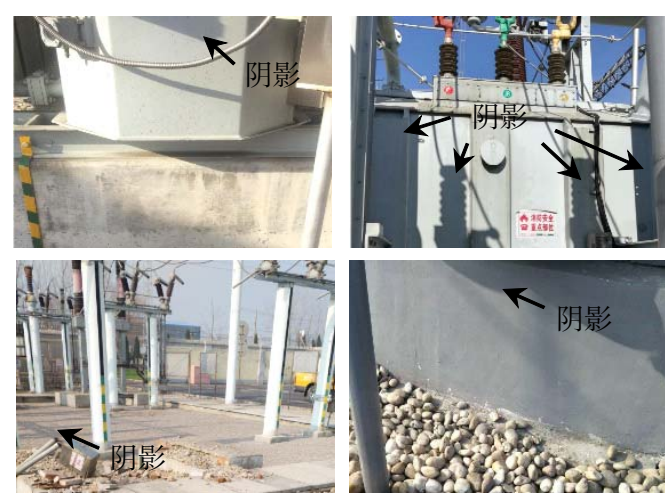

图 8 部分负样本图像

\section{4 实 验}

\section{1 数据预处理}

本文使用来源于电力系统中实际采集的数据, 共计地面油污图像 519 幅, 以 4:1 的比例随机划分 为训练集和测试集. 借助于开源的数据标注工具 LabelImg, 将标注的矩形框生成为可扩展标记语 言(extensible markup language, XML)文本文件，该 XML 文件类似于 Pascal VOC 数据集中目标检测的 标注结果. 负样本的 XML 文件不包含目标位置的 节点，只包含文件名、文件路径、图像宽度、高度 和通道数等信息.

在训练阶段, 首先对图像进行数据增强以提 高模型的泛化能力，包括随机裁剪、随机水平翻 转、随机垂直翻转等; 再以最长边为基准补零至长 宽相等，调整至 $600 \times 600$ 大小，并进行像素值的归 一化和标准化. 在测试阶段, 将图像以最长边为基 准补零至长宽相等, 调整至 $600 \times 600$ 大小，再进行 像素值的归一化与标准化.

\section{2 网络训练环境及参数设置}

实验均在 NVIDIA GTX 1080Ti GPU 配置的 Ubuntu16.04.1 系统上完成, 使用 PyTorch 深度学习
框架. 为了避免主网络初始化的权重给实验结果 造成不确定性影响, FPN 的主网络均采用在 ImageNet ${ }^{[17]}$ 预先训练过的 ResNet-50, 采用随机梯 度下降法进行模型参数的更新. 在使用循环训练 法时, 设网络开始进行困难样本挖掘训练次数 $S=50$, 距上一次困难样本挖掘后网络训练次数 $L=30$.

本文实验参数设置如表 1 所示.

表 1 实验参数表

\begin{tabular}{lc}
\hline \multicolumn{1}{c}{ 参数名 } & 参数值 \\
\hline epoch & 250 \\
batch size & 8 \\
learning rate & 0.001 \\
weight decay & 0.0005 \\
momentum & 0.9 \\
\hline
\end{tabular}

\section{3 实验结果及分析}

针对变压器漏油油污检测目标, 本文使用查 准率 $P$ 、查全率 $R$ 、平均准确率(average precision, AP)作为模型的衡量标准. 查准率表示模型预测出 的样本中真正样本的比例, 查全率为模型预测出 的真正样本占所有正样本的比例. 以查准率为纵 轴、查全率为横轴作图, 得到了查准率-查全率曲 线, 简称“PR 曲线”, $\mathrm{AP}$ 为曲线与坐标轴围成区域 的面积. 查准率和查全率的计算公式分别为

$$
\begin{aligned}
& P=\frac{\mathrm{TP}}{\mathrm{TP}+\mathrm{FP}}, \\
& R=\frac{\mathrm{TP}}{\mathrm{TP}+\mathrm{FN}} .
\end{aligned}
$$

为了验证循环训练、综合测试和负样本图像引 人等方法的有效性，设计了 8 组方案并进行实验: (1) 常规训练法和常规测试法(baseline); (2) 训练 时直接添加直方图均衡化样本 (hist eq); (3) 训练 时直接添加直方图均衡化样本并使用综合测试法 (hist eq+syn); (4) 循环训练法和常规测试法(loop); (5) 循环训练法和综合测试法(loop+syn); (6) 常规 训练法、常规测试法和负样本图像引入(neg); (7) 循环训练法、常规测试法和负样本图像引人 (neg+loop); (8) 循环训练法、综合测试法和负样本 图像引人 (neg+loop+syn). 最终的实验结果如表 2 所示, 模型的 LOSS 曲线和 PR 曲线分别如图 9 和 图 10 所示.

由表 2 可知, 使用原始数据集并以常规训练方 法和常规测试法进行训练, 模型的平均准确率、查 准率、查全率分别为 $52.54 \%, 78.26 \%, 58.44 \%$. 对 
表 2 实验结果

\begin{tabular}{lccc}
\hline \multicolumn{1}{c}{ 方案 } & AP & $P / \%$ & $R / \%$ \\
\hline baseline & 52.54 & 78.26 & 58.44 \\
hist_eq & 53.04 & 73.07 & 61.68 \\
hist_eq+syn & 53.91 & 73.48 & 62.99 \\
loop & 64.27 & 83.87 & 67.53 \\
loop+syn & 69.46 & 86.61 & 72.08 \\
neg & 63.63 & 81.88 & 67.53 \\
neg+loop & 66.23 & 85.15 & 70.78 \\
neg+loop+syn & $\mathbf{7 0 . 0 1}$ & $\mathbf{8 8 . 3 7}$ & $\mathbf{7 4 . 0 6}$ \\
\hline
\end{tabular}

注. 粗体表示实验的最优结果.

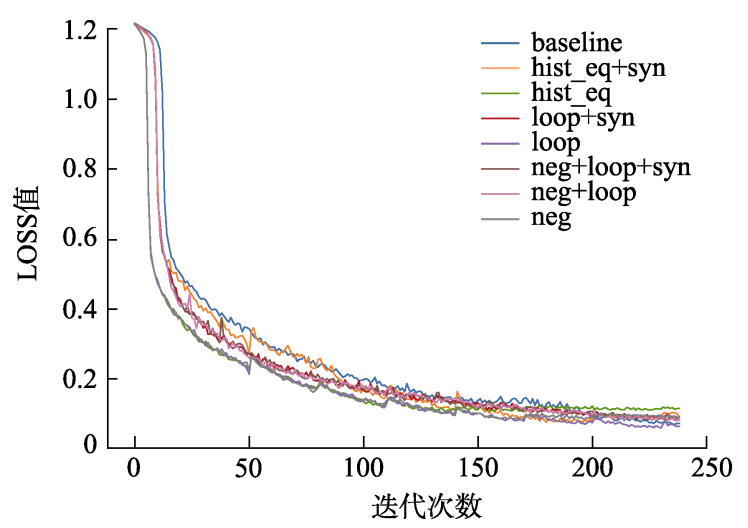

图 9 LOSS 曲线

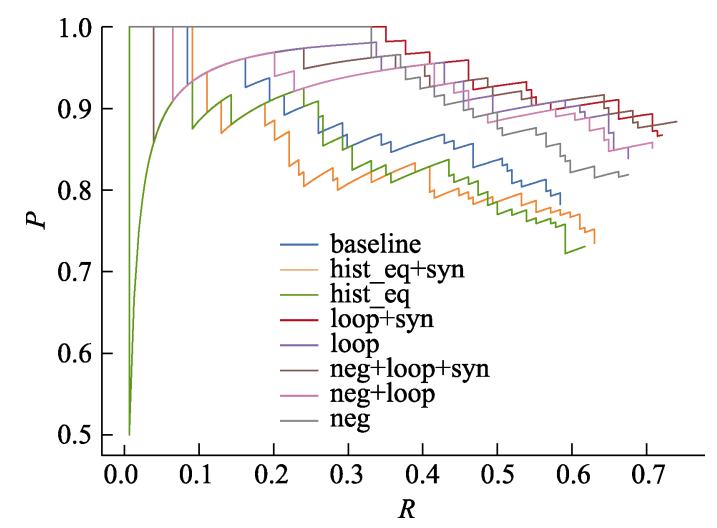

图 10 PR 曲线

比方案(1)(2)的实验结果, 直接添加均衡化后的样 本并训练模型, 模型的泛化性能无较大的提升, 但 查准率有一定的下降, 可能某些均衡化后的样本 对模型产生了一些未知的影响.

采用循环训练法后, 挖掘困难样本进行均衡 化增强后并再次训练, 提升了模型对困难样本的 泛化性能. 对比方案(1)(4)和方案(6)(7)的实验结 果, 油污的平均准确率分别提升 $11.73 \%, 2.6 \%$. 采 用综合测试法后, 对比方案(4)(5)和方案(7)(8)的实 验结果, 油污的平均准确率分别提升 $5.19 \%$, $3.84 \%$. 引人负样本后, 对比方案(1)(6)的实验结
果, 油污的平均准确率提升 $11.09 \%$, 这表明训练 负样本可以有效地缓解阴影误检的问题. 根据以 上的实验结果, 模型的平均准确率、查准率、查全 率与 baseline 相比分别提升 $17.47 \%, 10.11 \%$ 和 $15.56 \%$.

图 11 所示为方案(1)(5)模型预测结果对比, 图 11a 所示为方案(1)模型预测结果, 模型无法正确地 识别阴影与油污, 只定位到油污的部分区域. 图 $11 \mathrm{~b}$ 所示为方案 (5)模型预测结果. 使用循环训练法 和综合测试法后, 增大了油污与阴影之间的对比 度, 网络学习了更多的油污特征, 油污可以被正确 地检出. 由图 11 可见, 与使用常规训练和测试法 后的模型相比, 使用循环训练法和综合测试法预 测结果对于油污的定位更加准确.
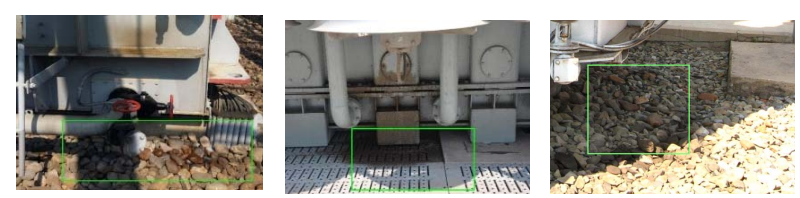

a. 方案(1)
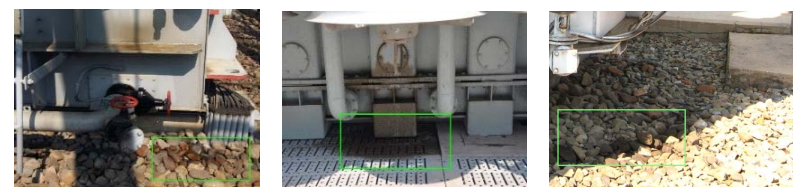

b. 方案 (5)

图 11 方案(1)(5)的模型预测结果对比

图 12 所示为方案(1)(6)模型预测结果对比. 图 $12 \mathrm{a}$ 所示为方案(1)模型预测结果, 对于一些明显的 阴影区域, 模型可以准确预测, 但图像中的一些单 独的阴影易被识别为油污. 图 $12 \mathrm{~b}$ 所示为方案(6) 模型预测结果, 在引人了负样本之后, 增强了模型 对阴影的识别能力, 其中的结果剔除了图 12a 被误 检的阴影区域, 使得油污的检测更加准确, 提高了 油污的查准率.
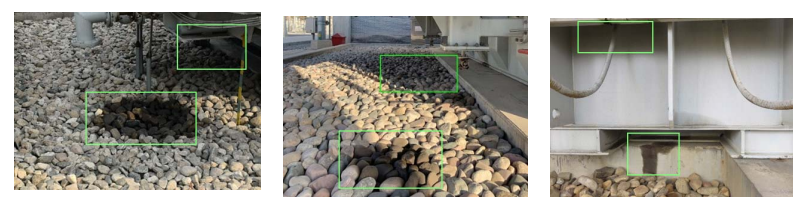

a. 方案(1)
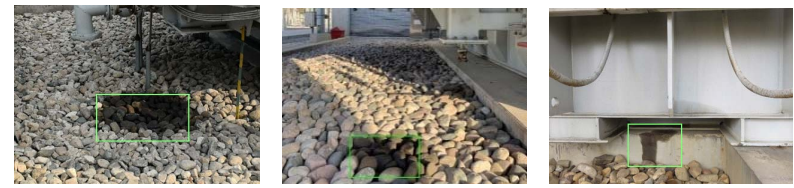

b. 方案(6)

图 12 方案(1)(6)模型预测结果对比 


\section{4 可视化结果分析}

为了验证提出的方法是否能使得网络学习到 关键特征, 本文可视化 RetinaNet 分类子网络的最 后一层特征图, 用以显示高层次特征的抽象表示. 在本文中, 模型只预测一个类别，因此最后一层特 征图具有 9 个通道, 共输出 9 张特征图. 图 13 和图 14 所示分别为方案(1)(5)模型和方案(1)(6)模型可 视化结果对比. 其中, ReLU 函数将特征图中所有 小于 0 的信息剔除，可视化后在图中呈现深紫色; 亮度较大的部分代表激活程度较大的区域, 亮度 较小的部分激活程度较小。

通过观察对比方案(1)(5)模型可视化结果，因 存在阴影覆盖在油污上, 方案(1)模型输出的特征 图中激活区域较大, 油污无法被准确激活. 使用循 环训练法训练模型后, 增强了网络对油污的特征 的提取能力, 因此方案(5)模型输出的特征图中的 激活区域较小，油污可以被准确定位.
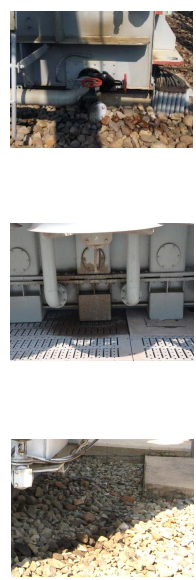

a. 测试图像

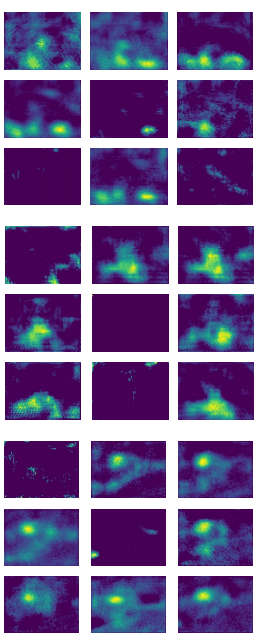

b. 方案(1)

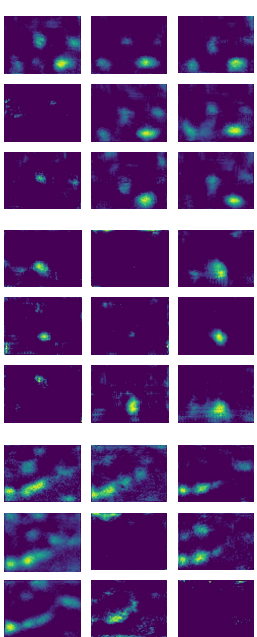

c. 方案(5)
图 13 方案(1)(5)的模型可视化结果

通过观察对比方案(1)(6)模型可视化结果，方 案(1)模型可以正确地检出一些无阴影覆盖干扰的 油污区域; 对于一些单独存在的阴影干扰, 无法有 效地区分阴影与油污，因此会存在阴影被误检测 成油污的情况. 对应至相应的特征图, 特征图中会 有多个不同的特征激活区域，如图 14b 所示. 方案 (6)模型在引人负样本之后, 特征图中的阴影激活 区域可以被有效地抑制，而油污的激活区域没有 发生改变, 如图 14c 所示.

\section{5 结 语}

针对变压器漏油油污检测问题，本文利用深

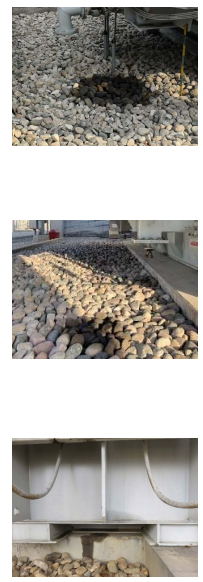

a. 测试图像

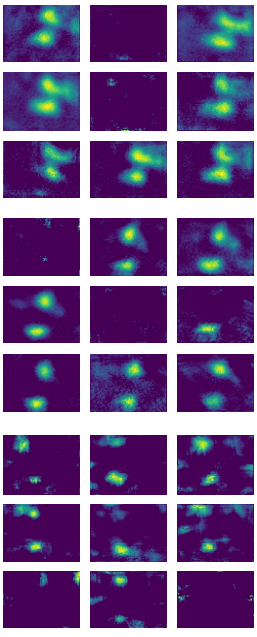

b. 方案(1)

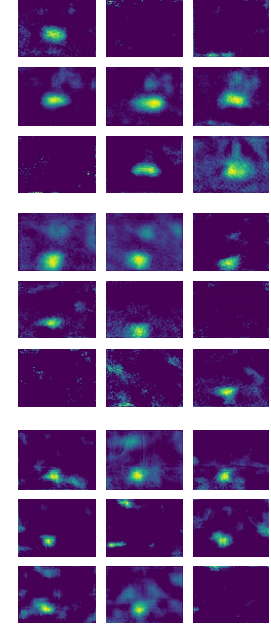

c. 方案(6)
图 14 方案(1)(6)的模型可视化结果

度学习算法并在其基础之上提出了一种循环训练 方法. 该方法循环地训练直方图均衡化后的困难 样本, 有助于模型学习到更多的油污特征, 提高了 模型对困难样本的学习能力, 缓解了由于光照阴 影对变压器漏油检测造成漏检问题, 增强了模型 对油污目标的定位与识别能力. 同时, 本文通过引 人负样本图像降低了模型的误检率, 减少了油污 的误检情况. 实验结果表明, 本文方法可以有效地 提高模型对漏油故障检测精确度, 消除了光照阴 影对漏油检测的影响, 平均准确率、查准率、查全 率均有大幅度提升.

\section{参考文献(References):}

[1] Dong Baoguo. Detection of transformer oil leakage based on image processing $[\mathrm{J}]$. Electric Power Construction, 2013, 34(11): 121-124(in Chinese)

(董保国. 基于图像处理的变压器渗漏油检测 $[\mathrm{J}]$. 电力建设, 2013, 34(11): 121-124)

[2] Li Gang, Yu Changhai, Liu Yunpeng, et al. Challenges and prospects of fault prognostic and health management for power transformer[J]. Automation of Electric Power Systems, 2017, 41(23): 156-167(in Chinese)

(李刚, 于长海, 刘云鹏, 等. 电力变压器故障预测与健康管 理: 挑战与展望[J]. 电力系统自动化, 2017, 41(23): 156-167)

[3] Liu Wei, Huang Shu, Ma Kai, et al. Application of video monitoring system in power system[J]. Guangdong Electric Power, 2014, 27(4): 57-60(in Chinese)

(刘玮，黄曙，马凯，等. 视频监控技术在电力系统中的应用 [J]. 广东电力, 2014, 27(4): 57-60)

[4] Krizhevsky A, Sutskever I, Hinton G E. ImageNet classification with deep convolutional neural networks $[\mathrm{J}]$. Communications of the ACM, 2017, 60(6): 84-90

[5] Girshick R. Fast R-CNN[C] //Proceedings of the IEEE Interna- 
tional Conference on Computer Vision. Los Alamitos: IEEE Computer Society Press, 2015: 1440-1448

[6] Li Yingtao, Xu Dan. Deep learning algorithm for woodcut prints style transfer[J]. Journal of Computer-Aided Design \& Computer Graphics, 2020, 32(11): 1804-1812(in Chinese) (李应涛, 徐丹. 木刻版画风格转换的深度学习算法 [J]. 计 算机辅助设计与图形学学报, 2020, 32(11): 1804-1812)

[7] Liu T R, Stathaki T. Faster R-CNN for robust pedestrian detection using semantic segmentation network[J]. Frontiers in Neurorobotics, 2018, 12: Article No.64

[8] Chen Chen, Huang Chao, Sun Song, et al. Vehicle detection algorithm based on multiple-model fusion[J]. Journal of Computer-Aided Design \& Computer Graphics, 2018, 30(11): 2134-2140(in Chinese)

(陈辰, 黄昆, 孙松，等. 多模型融合车辆检测算法 $[\mathrm{J}]$. 计算 机辅助设计与图形学学报, 2018, 30(11): 2134-2140)

[9] Litjens G, Kooi T, Bejnordi B E, et al. A survey on deep learning in medical image analysis[J]. Medical Image Analysis, 2017, 42: 60-88

[10] Girshick R, Donahue J, Darrell T, et al. Rich feature hierarchies for accurate object detection and semantic segmentation[C] // Proceedings of the IEEE Conference on Computer Vision and Pattern Recognition. Los Alamitos: IEEE Computer Society Press, 2014: 580-587

[11] Ren S Q, He K M, Girshick R, et al. Faster R-CNN: towards real-time object detection with region proposal networks[J]. IEEE Transactions on Pattern Analysis and Machine Intelligence, 2017, 39(6): 1137-1149

[12] Redmon J, Divvala S, Girshick R, et al. You only look once: unified, real-time object detection[C] //Proceedings of the IEEE Conference on Computer Vision and Pattern Recognition. Los Alamitos: IEEE Computer Society Press, 2016: 779-788

[13] Liu W, Anguelov D, Erhan D, et al. SSD: single shot multibox detector[C] //Proceedings of European Conference on Computer Vision. Heidelberg: Springer, 2016: 21-37
[14] Lin T Y, Goyal P, Girshick R, et al. Focal loss for dense object detection[C] //Proceedings of the IEEE International Conference on Computer Vision. Los Alamitos: IEEE Computer Society Press, 2017: 2999-3007

[15] Lin T Y, Maire M, Belongie S, et al. Microsoft COCO: common objects in context[C] //Proceedings of European Conference on Computer Vision. Heidelberg: Springer, 2014: 740-755

[16] Everingham M, van Gool L, Williams C K I, et al. The PASCAL visual object classes (VOC) challenge[J]. International Journal of Computer Vision, 2010, 88(2): 303-338

[17] He K M, Zhang X Y, Ren S Q, et al. Deep residual learning for image recognition[C] //Proceedings of the IEEE Conference on Computer Vision and Pattern Recognition. Los Alamitos: IEEE Computer Society Press, 2016: 770-778

[18] Lin T Y, Dollár P, Girshick R, et al. Feature pyramid networks for object detection[C] //Proceedings of the IEEE Conference on Computer Vision and Pattern Recognition. Los Alamitos: IEEE Computer Society Press, 2017: 936-944

[19] Shrivastava A, Gupta A, Girshick R. Training region-based object detectors with online hard example mining[C] // Proceedings of the IEEE Conference on Computer Vision and Pattern Recognition. Los Alamitos: IEEE Computer Society Press, 2016: 761-769

[20] Li X X, Liu Z W, Luo P, et al. Not all pixels are equal: difficulty-aware semantic segmentation via deep layer cascade[C] // Proceedings of the IEEE Conference on Computer Vision and Pattern Recognition. Los Alamitos: IEEE Computer Society Press, 2017: 3193-3202

[21] Hum Y C, Lai K W, Mohamad Salim M I. Multiobjectives bihistogram equalization for image contrast enhancement[J]. Complexity, 2014, 20(2): 22-36

[22] Szegedy C, Vanhoucke V, Ioffe S, et al. Rethinking the inception architecture for computer vision[C] //Proceedings of the IEEE Conference on Computer Vision and Pattern Recognition. Los Alamitos: IEEE Computer Society Press, 2016: 2818-2826 